\title{
Development of a Checklist Tool to Assess the Quality of Skin Lesion Images Acquired by Consumers Using Sequential Mobile Teledermoscopy
}

\author{
Uyen Koh $^{a}$ Brigid Betz-Stablein ${ }^{a}$ Montana O'Hara ${ }^{b}$ Caitlin Horsham ${ }^{b}$ \\ Clara Curiel-Lewandrowskic H. Peter Soyer ${ }^{\mathrm{a}}{ }^{\mathrm{d}}$ Monika Janda ${ }^{\mathrm{b}}$ \\ a The University of Queensland Diamantina Institute, The University of Queensland, Dermatology Research Centre, \\ Brisbane, QLD, Australia; ${ }^{b}$ Centre of Health Services Research, Faculty of Medicine, The University of Queensland, \\ Brisbane, QLD, Australia; ' ${ }^{\circ}$ epartment of Dermatology and the University of Arizona Cancer Center Skin Cancer \\ Institute, University of Arizona, Tucson, AZ, USA; ${ }^{\mathrm{d}}$ Department of Dermatology, Princess Alexandra Hospital, \\ Brisbane, QLD, Australia
}

\section{Keywords}

Melanocytic naevi $\cdot$ Melanoma $\cdot$ Skin cancer .

Skin self-examination - Telemedicine

\begin{abstract}
Background: Mobile teledermoscopy is an emerging technology that involves imaging and digitally sending dermoscopic images of skin lesions to a clinician for assessment. High-quality, consistent images are required for accurate telediagnoses when monitoring lesions over time. To date there are no tools to assess the quality of sequential images taken by consumers using mobile teledermoscopy. The purpose of this study was to develop a tool to assess the quality of images acquired by consumers. Methods: Participants imaged skin lesions that they felt were concerning at baseline, $1-$, and 2-months. A checklist to assess the quality of consumer sequential imaging of skin lesions was developed based on the International Skin Imaging Collaboration guidelines. A scale was implemented to grade the quality of the images: 0 (low) to 18 (very high). Intra- and inter-reliability of the checklist was assessed using Bland-Altman analysis. Using this checklist, the consistency with which 85 sets
\end{abstract}

karger@karger.com www.karger.com/drm

Karger $\stackrel{\text { ' }}{5}$

BOPEN ACCESS
(C) 2021 The Author(s)

Published by S. Karger AG, Basel

This is an Open Access article licensed under the Creative Commons Attribution-NonCommercial-4.0 International License (CC BY-NC) (http://www.karger.com/Services/OpenAccessLicense), applicable to the online version of the article only. Usage and distribution for commercial purposes requires written permission. of images were scored by 2 evaluators were compared using Kappa statistics. Items with a low Kappa value $<0.4$ were removed. Results: After reliability testing, 5 of the items were removed due to low Kappa values $(<0.4)$ and the final checklist included 13 items surveying: lesion selection; image orientation; lighting; field of view; focus and depth of view. Participants had a mean age of 41 years (range 19-73), and 67\% were female. Most participants ( $84 \%, n=71 / 85$ ) were able to select and image the correct lesion over time for both the dermoscopic and overview images. Younger participants (< 40 years old) scored significantly higher $(8.1 \pm 2.1)$ on the imaging checklist compared to older participants $(7.1 \pm 2.4$; $p=0.037)$. Participants had most difficulty with consistent image orientation. Conclusions: This checklist could be used as a triage tool to filter images acquired by consumers prior to telediagnosis evaluation, which would improve the efficiency and accuracy of teledermatology and teledermoscopy processes. It may also be used to provide feedback to the consumers to improve image acquisition over time.

(c) 2021 The Author(s)

Published by S. Karger AG, Basel

Part of the Nevi Article Series
Correspondence to:

Uyen Koh, u.koh@uq.edu.au 


\section{Introduction}

Advancements in mobile technologies have played a significant role in redefining the delivery of dermatological services to improve the diagnosis of melanoma or other skin cancers. Mobile teledermoscopy is one emerging technology that uses a dermatoscope attached to a smartphone to capture dermoscopic images of skin lesions via a mobile health application [1-6]. Mobile teledermoscopy may act as a communication tool between medical practitioners and patients for remote assessment. $\mathrm{Pa}$ tients can monitor a suspicious skin lesion identified by their medical practitioner or, alternatively, self-detect concerning lesions and forward images for remote medical assessment. A randomised, controlled trial comparing skin self-examination at home with and without mobile teledermoscopy confirmed the feasibility of mobile teledermoscopy and identified high sensitivity and specificity in detecting lesions suspicious for skin cancer in both arms [7-9]. Benefits of mobile teledermoscopy identified by consumers include the convenience of a remote service, shorter waiting times, and potential cost savings [8]. Consumers also reported a high acceptance of mobile teledermoscopy $[8,10]$.
One of the challenges encountered when consumers, rather than clinicians, conduct the imaging process is the quality of the images $[1,4]$. When using sequential imaging to monitor a lesion over time, additional challenges may impact the ease of telediagnoses. These include changes in image orientation, lighting, and focus that may alter the appearance of the lesion. Sequential dermoscopy imaging by clinicians has been shown to increase the sensitivity for melanoma diagnosis and reduce the benign to malignant excision ratio [11]. This is because photographic evidence can demonstrate lesion stability (or lack thereof) to minimise the need for unnecessary biopsies. Standardisation is important to obtain accurate telediagnoses and guidelines have been proposed to standardise dermatological images taken by clinicians. The International Skin Imaging Collaboration (ISIC) established a set of 9 criteria to ensure images taken by clinicians are of sufficient quality for diagnoses. These include guidance on optimal lighting, background colour, field of view, and image orientation, among others [12]. Due to the recent shift of consumers conducting their own imaging and a higher dependence on telehealth services during the COVID-19 pandemic, a tool is required to assess the image quality of consumer-imaged skin lesions [13].
Fig. 1. A set of dermoscopic and overview images taken of a skin lesion at baseline, 1, and 2 months.

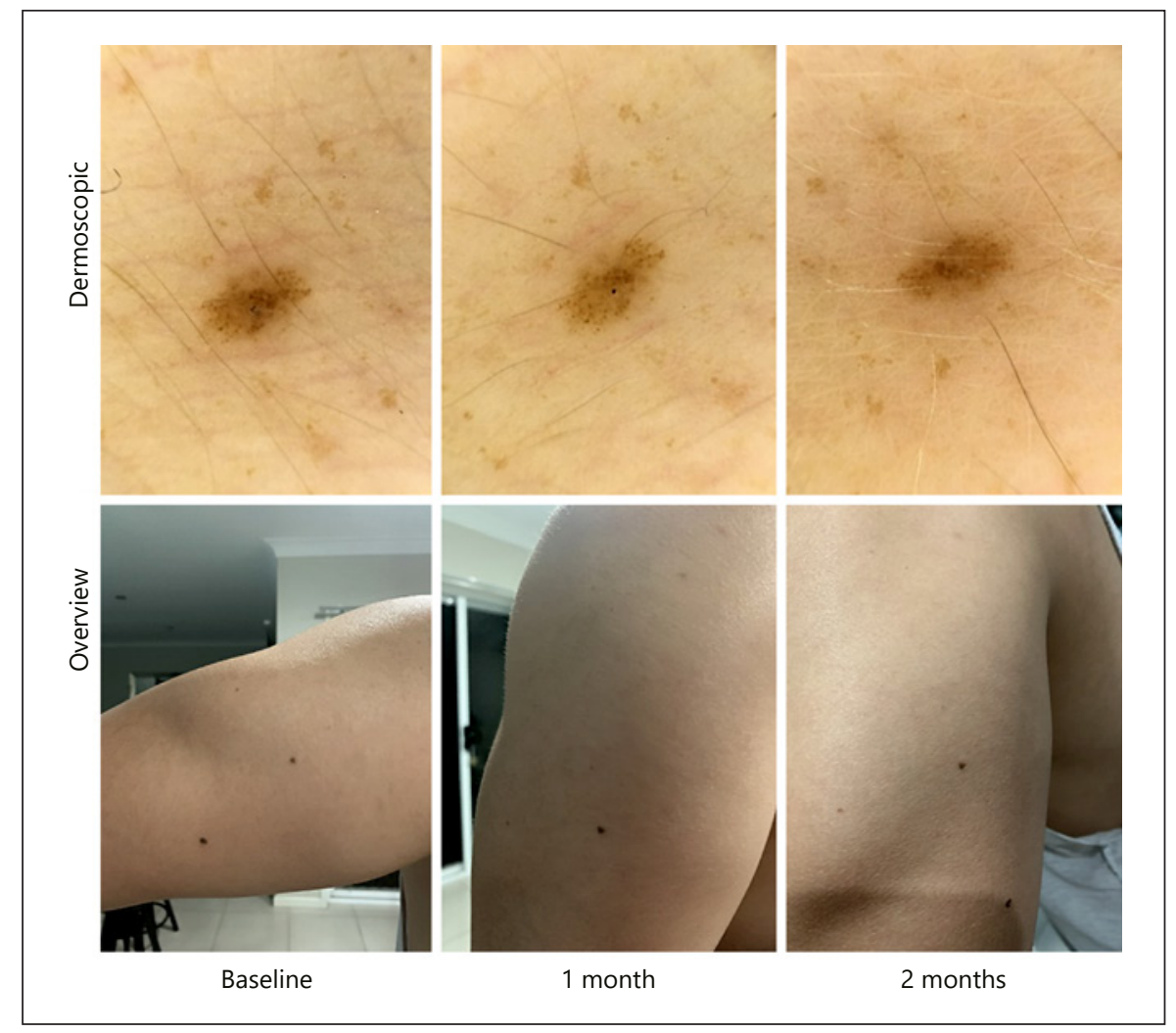


We developed and tested the reliability of a checklist adapted from ISIC guidelines to score the image quality of lesions longitudinally imaged by consumers. The checklist was assessed using data from study participants who conducted consumer-led mobile teledermoscopy for the early detection of skin cancer.

\section{Materials and Methods}

The images used for this study were acquired from participants in the mobile teledermoscopy arm of a randomised, controlled trial that compared mobile teledermoscopy-enhanced skin self-examinations to naked-eye skin self-examinations. The methods and the main outcomes of the trial, including sensitivity and specificity of skin cancer detection, have been previously reported [14, 15]. Ethics approval was obtained from the Queensland University of Technology Human Research Ethics Committee (approval No. 1400000807). Briefly, the intervention group was advised to use mobile teledermoscopy to conduct 3 skin self-examinations at home, each 1 month apart. Participants were asked to image skin lesions that they felt were suspicious for skin cancer using their own smartphone, a mobile dermatoscope, and corresponding Handyscope application (app; FotoFinder System GmbH, Bad Birnbach, Germany). Participants were provided with written instructions and a video on how to use the dermatoscope and app, how to detect lesions using the asymmetry and colour rule [16], and how to conduct a whole-body skin self-examination. Participants then presented for an in-person whole-body skin examination by a dermatologist after completing their third self-examina-

Table 1. Proposed checklist to assess the quality and standardisation of consumer sequential imaging of pigmented lesions

\begin{tabular}{|c|c|c|c|}
\hline & 1 & 0 & Description $^{1}$ \\
\hline \multicolumn{4}{|l|}{ Lesion selection } \\
\hline 1. Was the same lesion dermoscopically imaged at each timepoint? & Yes & No & \multirow[t]{2}{*}{ Ensure the patient photographed the same lesion } \\
\hline 2. Was the same lesion imaged in the overview photographs at each timepoint? & Yes & No & \\
\hline 3. Can you tell which lesion was dermoscopically imaged on the overview images? & Yes & No & $\begin{array}{l}\text { If there are multiple lesions in the overview image, it } \\
\text { must be clear which is the lesion being examined }\end{array}$ \\
\hline 4. Can you clearly identify where the lesion of interest is located on the body? & Yes & No & $\begin{array}{l}\text { The overview image should identify which area on the } \\
\text { body the lesion is located }\end{array}$ \\
\hline \multicolumn{4}{|l|}{ Image orientation } \\
\hline 5. Was the orientation of the dermoscopic images consistent over time? & Yes & No & \multirow{2}{*}{$\begin{array}{l}\text { The camera should be positioned in the same } \\
\text { orientation (such as asking the user to hold the } \\
\text { camera horizontally each time) }\end{array}$} \\
\hline 6. Was the orientation of the overview images consistent over time? & Yes & No & \\
\hline \multicolumn{4}{|l|}{ Lighting for overview images } \\
\hline 7. Was the background lighting adequate to view the lesion at timepoint 1 ? & Yes & No & \multirow{4}{*}{$\begin{array}{l}\text { Using natural lighting is best for overview images; but } \\
\text { broad-spectrum lighting (rather than fluorescent } \\
\text { lighting) may be used. Even lighting will reduce } \\
\text { shadows }\end{array}$} \\
\hline 8. Was the background lighting adequate to view the lesion at timepoint 2 ? & Yes & No & \\
\hline 9. Was the background lighting adequate to view the lesion at timepoint 3 ? & Yes & No & \\
\hline 10. Was the background lighting consistent over time? & Yes & No & \\
\hline \multicolumn{4}{|l|}{ Background colour for overview images } \\
\hline 11. There was no reflection from objects in the background of an image & True & False & \multirow{2}{*}{$\begin{array}{l}\text { A neutral coloured, plain background should be used } \\
\text { with no objects or distractors. Reflection of objects } \\
\text { may change skin colour appearance }\end{array}$} \\
\hline 12. Absence of jewellery or other distracters in the images & True & False & \\
\hline \multicolumn{4}{|l|}{ Field of view for dermoscopic images } \\
\hline 13. Is Lesion 1 in the centre of the dermoscopic image? & Yes & No & \multirow{3}{*}{$\begin{array}{l}\text { The lesion should be in the centre of the image, so the } \\
\text { surrounding skin can be viewed with equal areas of } \\
\text { surrounding skin at the periphery }\end{array}$} \\
\hline 14. Is Lesion 2 in the centre of the dermoscopic image? & Yes & No & \\
\hline 15. Is Lesion 3 in the centre of the dermoscopic image? & Yes & No & \\
\hline \multicolumn{4}{|l|}{ Focus and depth of field for dermoscopic images } \\
\hline 16. Based on the focus, were you able to evaluate the lesion at timepoint 1 ? & Yes & No & \multirow{3}{*}{$\begin{array}{l}\text { The lesion, including the surrounding skin, should be } \\
\text { in focus with no blurriness }\end{array}$} \\
\hline 17. Based on the focus, were you able to evaluate the lesion at timepoint 2 ? & Yes & No & \\
\hline 18. Based on the focus, were you able to evaluate the lesion at timepoint 3 ? & Yes & No & \\
\hline
\end{tabular}


tion. Figure 1 shows an example of a set of dermoscopic and overview images of a skin lesion taken at the 3 timepoints.

\section{Development of Consumer Image Quality Checklist}

To assess the quality of the sequential imaging of pigmented skin lesions acquired by consumers, a checklist (Table 1) was developed based on the Standards for Dermatological Imaging established by the ISIC [12]. The guidelines were adapted to focus on the quality and standardisation of sequential images taken by consumers as opposed to health professionals.

Four domains from the ISIC recommendations were not relevant for imaging conducted by consumers using smartphones. The app used in this study automatically controls resolution (i.e., the number of pixels); therefore, resolution was not included in this checklist. The app does not have a digital measurement tool and thus the "scale and measuring using digital imaging software" domain was excluded from this checklist. The "image storage" and "colour calibration" domains were excluded from the checklist as these were not relevant to consumer imaging. Two dermatologists (H.P.S. and C.C.-L.) assessed the content of the checklist and amended the checklist accordingly until both were in agreement that the remaining items were relevant.

\section{Checklist Scoring}

The initial checklist consisted of 18 items that were scored either as of unacceptable or acceptable quality for telediagnosis receiving a value of " 0 " or " 1 ," respectively. The items were then summed for a maximum score of 18 , with higher scores indicating better quality. Sub-scores were also calculated for each of the domains suggested by the ISIC, as per Table 1 ("lesion selection," "image orientation," "lighting for overview images," "background colour for overview images," "field of view for dermoscopic images," and "focus and depth of field for dermoscopic images").

\section{Sample Size Calculation}

To estimate the mean quality of images within \pm 1.1 units with $95 \%$ confidence assuming an SD of 2.8 , a total sample size of 85 evaluated sets of images were required. Bland-Altman analysis was used to calculate $95 \%$ limits of agreement for both inter and intrarater reliability. Assuming an SD of 0.95, a total of 36 sets of images needed to be scored by 2 evaluators to calculate the $95 \%$ limits of agreement with a precision of \pm 0.5 .

\section{Assessment of Images}

Participants submitted a median of 6 lesions at each skin selfexamination (range 1-24). To ensure the analysis was not biased, a computer-generated list of the images from all participants was used to randomly select and assess one image set per participant.

Two researchers (U.K. and M.O.) assessed the quality of the sequential sets of dermoscopic and overview images taken by 85 participants. Each rater independently assessed a total of 71 sets of images, with 36 image sets overlapping between the 2 evaluators for the inter-rater reliability assessment. Both evaluators also assessed the 36 overlapping sets of images twice on different days at least 2 weeks apart to establish intra-rater reliability.

\section{Statistical Analysis}

The inter- and intra-rater reliability of each of the individual 18 checklist items was assessed using Cohen's kappa, with kappa values of $0.01-0.20,0.21-0.40,0.41-0.60,0.61-0.80$, and $0.81-1.00$
Table 2. Demographics $(n=85)$

\begin{tabular}{|c|c|c|}
\hline & $n(\%)$ & $p$ value \\
\hline Age & & $0.037^{*}$ \\
\hline 40 years & $45(53)$ & \\
\hline$>40$ years & $40(47)$ & \\
\hline Sex & & 0.502 \\
\hline Female & $57(67)$ & \\
\hline Male & $28(33)$ & \\
\hline Relationship status & & 0.999 \\
\hline Living with partner & $67(79)$ & \\
\hline Living without partner & $18(21)$ & \\
\hline Highest education level & & 0.097 \\
\hline University degree/diploma & $64(75)$ & \\
\hline Trade or technical certificate & $15(18)$ & \\
\hline Secondary school & $6(7)$ & \\
\hline Employment status & & 0.907 \\
\hline Full-time (including self-employed) & $55(65)$ & \\
\hline Part-time or casual & $27(32)$ & \\
\hline Student & 0 & \\
\hline Retired & 0 & \\
\hline Other & $3(3)$ & \\
\hline Previous skin spot or mole excised or treated & & 0.591 \\
\hline Yes & $53(62)$ & \\
\hline No or unsure & $32(38)$ & \\
\hline Skin type & & 0.337 \\
\hline Very fair & $74(87)$ & \\
\hline Medium & $10(12)$ & \\
\hline Dark & $1(1)$ & \\
\hline Self-reported naevus density & & 0.840 \\
\hline None & 0 & \\
\hline Few $(<20)$ & $25(29)$ & \\
\hline Some $(20-50)$ & $34(40)$ & \\
\hline Many $(>50)$ & $26(31)$ & \\
\hline
\end{tabular}

* Statistically significant.

indicating none to slight, fair, moderate, substantial, and almost perfect agreement, respectively. Items with a Cohen's kappa of $<0.4$ were removed from the final checklist due to poor inter-rater reliability. Inter- and intra-rater agreement of the overall image quality score was assessed using Bland-Altman analysis. Student $t$ tests and analysis of variance were used to assess whether participant characteristics were associated with overall image quality score.

\section{Results}

Eighty-five participants provided images at all 3 timepoints and remained for the analysis. The participants' mean age was 41 years, they were mostly female $(67.1 \%$, $n=57 / 85)$, and had a university degree/diploma $(75.3 \%$, $n=64 / 85$; Table 2). 
Table 3. Individual question scores and Cohen's kappa

\begin{tabular}{|c|c|c|c|}
\hline Question & $\begin{array}{l}\text { Yes, } \\
n(\%)\end{array}$ & $\begin{array}{l}\text { No, } \\
n(\%)\end{array}$ & $\begin{array}{l}\text { Cohen's } \\
\text { kappa }\end{array}$ \\
\hline \multicolumn{4}{|l|}{ Lesion selection } \\
\hline 1. Same lesion (dermoscopic) & $71(84)$ & $14(16)$ & 0.89 \\
\hline 2. Same lesion (overview) & $71(84)$ & $14(16)$ & 0.65 \\
\hline 3. ID lesion on overview & $45(53)$ & $40(47)$ & 0.49 \\
\hline 4. Clearly ID lesion location & $54(64)$ & $31(36)$ & 0.83 \\
\hline \multicolumn{4}{|l|}{ Image orientation } \\
\hline 5. Same orientation (dermoscopic) & $38(45)$ & $47(55)$ & 0.55 \\
\hline 6. Same orientation (overview) & $33(39)$ & $52(61)$ & 0.66 \\
\hline \multicolumn{4}{|l|}{ Lighting for overview images } \\
\hline 7. Background lighting timepoint 1 & $39(46)$ & $46(54)$ & 0.30 \\
\hline 8. Background lighting timepoint 2 & $31(36)$ & $54(64)$ & 0.35 \\
\hline 9. Background lighting timepoint 3 & $24(28)$ & $61(72)$ & 0.15 \\
\hline 10. Background lighting consistent over time & $64(75)$ & $21(25)$ & 0.61 \\
\hline \multicolumn{4}{|l|}{ Background colour for overview images } \\
\hline 11. No reflection & $38(45)$ & $47(55)$ & 0.36 \\
\hline 12. No jewellery or distracters & $74(87)$ & $11(13)$ & 0.30 \\
\hline \multicolumn{4}{|l|}{ Field of view for dermoscopic images } \\
\hline 13. Lesion 1 at centre of dermoscopic image & $49(58)$ & $36(42)$ & 0.68 \\
\hline 14. Lesion 2 at centre of dermoscopic image & $40(47)$ & $45(53)$ & 0.51 \\
\hline 15. Lesion 3 at centre of dermoscopic image & $39(46)$ & $46(54)$ & 0.56 \\
\hline \multicolumn{4}{|l|}{ Focus and depth of field for dermoscopic images } \\
\hline 16. Timepoint 1 adequately focused for evaluation & $60(71)$ & $25(29)$ & 0.48 \\
\hline 17. Timepoint 2 adequately focused for evaluation & $69(81)$ & $16(19)$ & 0.58 \\
\hline 18. Timepoint 3 adequately focused for evaluation & $57(67)$ & $28(33)$ & 0.50 \\
\hline
\end{tabular}

Italicised values indicate slight or fair inter-rater reliability.

\section{Reliability of Individual Checklist Items}

Cohen's kappa scores for 13 of 18 items on the checklist showed moderate to almost perfect inter-rater agreement between the 2 evaluators (Table 3 ). Cohen's kappa showed fair agreement for items 7 and 8 (lighting for overview images), 11 and 12 (background colour), and slight agreement for item 9 (lighting for overview images) between the 2 evaluators.

\section{Inter-and Intra-Rater Reliability Scores of the Overall Image Checklist}

Inter-rater assessment showed that $95 \%$ of the time the 36 image quality scores of the 2 evaluators were within \pm 3.61 of each other. Intra-rater reliability was assessed for both evaluators and the $95 \%$ limits of agreement showed high repeatability with image quality scores within \pm 1.7 and \pm 2.1 , respectively.

\section{Final Checklist}

The 5 items that had poor agreement based on Cohen's kappa calculations below 0.4 were removed. Once re- moved, $95 \%$ of the time the scores between the 2 evaluators were within \pm 2.74 of each other.

Intra-rater reliability was also reassessed after removing the 5 items that had poor agreement between the 2 raters. For Evaluator 1, 95\% of the time the scores between their 2 evaluations were within \pm 1.39 . For Evaluator 2 , the scores were within \pm 2.23 for $95 \%$ of the time between their 2 evaluations. Table 4 presents the 13-item final checklist; its score can range from 0 to 13 .

\section{Quality of Images}

Based on the 13-item checklist, participants had a mean overall image quality score of $7.6 \pm 2.3$ (range $2-12$ ). The domains that commonly performed well included "focus and depth of field for dermoscopic images" and "lesion selection." Domains that were poorly performed were the "lighting for the overview images" and "image orientation" (Table 5).

Most participants ( $84 \%, n=71 / 85)$ were able to select and re-image the correct lesion over time for both the dermoscopic and overview images. Over half of the par- 
Table 4. Final checklist after reliability testing to assess the quality and standardisation of consumer sequential imaging of pigmented lesions

1

Lesion selection

1. Was the same lesion dermoscopically imaged at each time point?

2. Was the same lesion imaged in the overview photographs at each time point?

3. Can you tell which lesion was dermoscopically imaged on the overview images?

4. Can you clearly identify where the lesion of interest is located on the body? Image orientation

5. Was the orientation of the dermoscopic images consistent over time?

6 . Was the orientation of the overview images consistent over time? Lighting for overview images

7. Was the background lighting consistent over time?

Field of view for dermoscopic images

8. Is Lesion 1 in the centre of the dermoscopic image?

9. Is Lesion 2 in the centre of the dermoscopic image?

10. Is Lesion 3 in the centre of the dermoscopic image?

Focus and depth of field for dermoscopic images

11. Based on the focus, were you able to evaluate lesion at timepoint 1 ?

12. Based on the focus, were you able to evaluate lesion at timepoint 2?

13. Based on the focus, were you able to evaluate lesion at timepoint 3 ?

$\begin{array}{ll}\text { Yes } & \text { No } \\ \text { Yes } & \text { No } \\ \text { Yes } & \text { No } \\ \text { Yes } & \text { No } \\ & \\ \text { Yes } & \text { No } \\ \text { Yes } & \text { No } \\ & \\ \text { Yes } & \text { No } \\ & \\ \text { Yes } & \text { No } \\ \text { Yes } & \text { No } \\ \text { Yes } & \text { No } \\ & \\ \text { Yes } & \text { No } \\ \text { Yes } & \text { No } \\ \text { Yes } & \text { No }\end{array}$

ticipants had dermoscopic $(55 \%, n=47 / 85)$ and overview $(61 \%, n=52 / 85)$ images that were inconsistent in their orientation over time. Most of the images were in focus for evaluation at each of the 3 timepoints (Table 3 ).

\section{Demographic Associations}

Younger participants ( $<40$ years old) submitted higher quality images and achieved significantly higher scores $(8.1 \pm 2.1)$ compared to older participants $(7.1 \pm 2.4 ; p=$ 0.037 ; Table 2). No other demographic variables were significantly associated with the image quality scores.

\section{Discussion}

During COVID-19 an increase in the use of asynchronous teledermatology services has been observed globally [17]. Teledermatology has become a crucial method of continuing to deliver care during the peak of the pandemic, with all non-urgent in-person dermatology visits postponed. The quality of consumer-taken images is thus critical for the safe delivery of teledermatology services and sequential imaging should employ consistent imaging techniques to aid comparison of images over time [18]. There has been a steady increase in the number of mobile apps, even prior to COVID-19, that facilitate image capture and lesion tracking available for the general public to support the early detection of skin cancers, and
Table 5. Overall and domain scores (13-item checklist)

\begin{tabular}{lccc}
\hline & Mean & Range & $95 \%$ CI \\
\hline Overall score & 7.6 & $2-12$ & $7.1-8.1$ \\
$\begin{array}{l}\text { Domain } \\
\quad \text { Lesion selection }\end{array}$ & 2.8 & $0-4$ & $2.6-3.1$ \\
$\quad$ Image orientation & 0.8 & $0-2$ & $0.7-1.0$ \\
$\quad$ Lighting for overview images & 0.2 & $0-1$ & $0.2-0.3$ \\
$\quad$ Field of view for dermoscopic images & 1.5 & $0-3$ & $1.3-1.7$ \\
$\quad$ Focus and depth of field for & & & \\
$\quad$ dermoscopic images & 2.2 & $0-3$ & $2.0-2.3$ \\
\hline
\end{tabular}

dermatoscopes targeted at consumers have also become available [19]. However, despite an increased reliance on teledermatology, there are no imaging quality checklists for images taken outside of clinical practices particularly by non-experts [13, 20,21]. The lack of development, validation, and adoption of universal imaging practices may result in lack of consumer training, and high variation in image quality $[21,22]$. For example, if 2 images are taken of a lesion months apart with different orientation and lighting, this can jeopardise the ability of the clinician to detect relevant changes over time. Therefore, education and guidance for consumers to conduct reimaging in a standardised way based on the quality checklist developed here has strong potential to improve the accuracy of telediagnoses. 
In clinical practice we envisage that this checklist will also improve the workflow for clinicians. This checklist could be implemented as a pre-screening tool for medical assistants, whereby they screen the images for quality prior to forwarding the images to a clinician for interpretation. If the images fail to properly score in a quality checklist domain, the patient would be asked to repeat the imaging process and re-send the image. Such a system has the potential to save time and increase the efficiency of time-poor clinicians. Participants in this study were provided with imaging instructions; however, despite this attempt, some still struggled to capture images of the lesions correctly. Over half of participants did not have the same image orientation in at least one of their follow-up images. Further written and visual instructions on holding the camera horizontal to the skin surface in each image may be helpful. Consumers could also complete the quality checklist themselves before submitting their images. From the image sets evaluated in this study, a small proportion of images (5\%) were unsuitable for clinical assessment [15], indicating that consumers are usually capable of taking images that are acceptable; however, better quality could further improve the benefit of consumer-acquired teledermoscopy.

Three items on overview image lighting and 2 items on background colour were removed from the checklist due to poor inter-rater reliability. Rather than capturing lighting acceptability at each of the 3 timepoints, the final checklist contains a single item that asks for lighting consistency across the timepoints. Overview images are important as they provide an indication of lesion shape, size, and anatomic location, and aid lesion re-identification over time $[23,24]$. Consumers should capture overview images in the same light source to ensure consistency [25]. Inconsistent lighting can significantly challenge teledermatologists to provide a diagnosis, and we found the main inconsistency was images taken in natural and artificial light [26]. While using natural light is best for overview images, this is not always practical due to variability in daylight hours, seasonal changes, and weather conditions which cannot be controlled [12, 25]. Overall, our results indicate participants may have had more difficulty standardising the overview images, rather than the dermoscopic images. In this study, the participants were provided with a dermatoscope with in-built lighting which reduces the variability of dermoscopic lighting. However, not all dermatoscopes will have this feature. While reflection from objects in the background of an image may change the appearance of skin tone [12], this item was removed due to the poor inter-rater agreement, but perhaps should be reintroduced with a more stringent definition, including specifically indicating the absence of jewellery covering the lesion, absence of a shadow over the lesion, and so on, which may improve the inter-rater reliability of this domain.

The next step in the development of this checklist is to derive a cut-off score to determine if images are acceptable for a telediagnosis. This cut-off score will help medical assistants determine whether to forward the images to the dermatologist for assessment or back to the consumer to re-image the lesions.

\section{Limitations}

The volunteer sample may limit the generalisability of the results as participants were potentially more receptive to using mobile technologies [9]. Over half of the participants were female, well educated, and this study excluded participants without an iPhone. This study was also limited by a small sample size, and further testing is required in larger cohorts. This checklist was tested on skin lesions for the early detection of skin cancer only, but further testing could assess its reliability for imaging of other inflammatory skin diseases. This checklist was developed and tested for individuals who conducted imaging at 3 separate timepoints. The checklist may be adapted to assess images captured once only or at a different number of timepoints.

\section{Conclusions}

This image quality checklist includes the key aspects of dermatological imaging that in our view are paramount for standardisation of sequential imaging by consumers. With further validation on a larger sample and a defined cut-off score, this image quality checklist may be used in clinical practice to improve the workflow and determine if the image quality is sufficient to enable accurate telediagnoses.

\section{Key Message}

We developed a checklist to assist with standardisation of image quality in consumers.

\section{Statement of Ethics}

This study was approved by the Queensland University of Technology Human Ethics Committee (QUT approval No. 1400000807). 


\section{Conflict of Interest}

H.P.S. is a shareholder of MoleMap NZ Limited and e-derm consult $\mathrm{GmbH}$, and undertakes regular teledermatological reporting for both companies. H.P.S. is a Medical Consultant for Canfield Scientific Inc., Revenio Research Oy, and also a Medical Advisor for First Derm. C.C.-L. is an advisory board member for BMS and Helsinn Pharm.

\section{Funding Sources}

The study was funded by a research grant awarded to M.J. from the National Health and Medical Research Council (APP1113962). M.J. is funded by a TRIP Fellowship (APP1151021). HPS is funded by the Medical Research Future Fund - Next Generation Clin- ical Researcher's Program Practitioner Fellowship (APP1137127). C.C.-L. is partially funded by an NIH/NCI P01 CA27502 grant and the Alan and Janice Levin Endowed Chair in Cancer Research.

\section{Author Contributions}

U.K., H.P.S., and C.C.-L. created and assessed the checklist. U.K. and M.O. assessed the quality of the sequential sets of dermoscopic and overview images. C.H. and M.J. conducted the randomised, controlled trial data collection. U.K. and B.B.-S. conducted the statistical analysis. U.K., C.H., and M.O. participated in the drafting and writing of the manuscript. All authors contributed to the interpretation and critical revision of the manuscript. All authors provided final approval of this paper.

\section{References}

1 Massone C, Brunasso AM, Campbell TM, Soyer HP. Mobile teledermoscopy - melanoma diagnosis by one click? Semin Cutan Med Surg. 2009 Sep;28(3):203-5.

2 Massone C, Brunasso AM, Hofmann-Wellenhof R, Gulia A, Soyer HP. Teledermoscopy: education, discussion forums, teleconsulting and mobile teledermoscopy. G Ital Dermatol Venereol. 2010 Feb;145(1):127-32.

3 Lee KJ, Finnane A, Soyer HP. Recent trends in teledermatology and teledermoscopy. Dermatol Pract Concept. 2018 Jul;8(3):214-23.

4 Sunderland M, Teague R, Gale K, Rademaker M, Oakley A, Martin RC. E-referrals and teledermatoscopy grading for melanoma: a successful model of care. Australas J Dermatol. 2020 May;61(2):147-51.

5 Arzberger E, Curiel-Lewandrowski C, Blum A, Chubisov D, Oakley A, Rademaker M, et al. Teledermoscopy in high-risk melanoma patients: a comparative study of face-to-face and teledermatology visits. Acta Derm Venereol. 2016 Aug;96(6):779-83.

6 Congalton AT, Oakley AM, Rademaker M, Bramley D, Martin RC. Successful melanoma triage by a virtual lesion clinic (teledermatoscopy). J Eur Acad Dermatol Venereol. 2015 Dec;29(12):2423-8.

7 Manahan MN, Soyer HP, Loescher LJ, Horsham C, Vagenas D, Whiteman DC, et al. A pilot trial of mobile, patient-performed teledermoscopy. Br J Dermatol. 2015 Apr;172(4):1072-80.

8 Koh U, Horsham C, Soyer HP, Loescher LJ, Gillespie N, Vagenas D, et al. Consumer acceptance and expectations of a mobile health application to photograph skin lesions for early detection of melanoma. Dermatology. 2019;235(1):4-10.

9 Wu X, Oliveria SA, Yagerman S, Chen L, DeFazio J, Braun R, et al. Feasibility and efficacy of patient-initiated mobile teledermoscopy for short-term monitoring of clinically atypical nevi. JAMA Dermatol. 2015 May;151(5): 489-96.
10 Horsham C, Snoswell C, Vagenas D, Loescher LJ, Gillespie N, Soyer HP, et al. Is teledermoscopy ready to replace face-to-face examinations for the early detection of skin cancer? Consumer views, technology acceptance, and satisfaction with care. Dermatology. 2020; 236(2):90-6.

11 Adler NR, Kelly JW, Guitera P, Menzies SW, Chamberlain AJ, Fishburn P, et al. Methods of melanoma detection and of skin monitoring for individuals at high risk of melanoma: new Australian clinical practice. Med J Aust. 2019 Jan;210(1):41-7.

12 Finnane A, Curiel-Lewandrowski C, Wimberley G, Caffery L, Katragadda C, Halpern A, et al.; International Society of Digital Imaging of the Skin (ISDIS) for the International Skin Imaging Collaboration (ISIC). Proposed technical guidelines for the acquisition of clinical images of skin-related conditions. JAMA Dermatol. 2017 May;153(5):453-7.

13 Rat C, Hild S, Rault Sérandour J, et al. Use of smartphones for early detection of melanoma: systematic review. J Med Internet Res. 2018;20(4):e135.

14 Janda M, Horsham C, Koh U, Gillespie N, Loescher LJ, Vagenas D, et al. Redesigning skin cancer early detection and care using a new mobile health application: protocol of the SKIN research project, a randomised controlled trial. Dermatology. 2019;235(1):11-8.

15 Janda M, Horsham C, Vagenas D, Loescher LJ, Gillespie N, Koh U, et al. Accuracy of mobile digital teledermoscopy for skin self-examinations in adults at high risk of skin cancer: an open-label, randomised controlled trial. Lancet Digit Health. 2020 Mar; 2(3):e129-37.

16 Luttrell MJ, Hofmann-Wellenhof R, Fink-Puches R, Soyer HP. The AC Rule for melanoma: a simpler tool for the wider community. J Am Acad Dermatol. 2011 Dec;65(6):1233-4.
17 Su MY, Das S. Expansion of asynchronous teledermatology during the COVID-19 pandemic. J Am Acad Dermatol. 2020;83(6):e471e2.

18 Abbott LM, Miller R, Janda M, Bennett H, Taylor M, Arnold C, et al. Practice guidelines for teledermatology in Australia. Australas J Dermatol. 2020;61(3):e293-302.

19 Kong FW, Horsham C, Ngoo A, et al. Review of smartphone mobile applications for skin cancer detection: what are the changes in availability, functionality, and costs to users over time? Int J Dermatol. 2020, Online ahead of print.

20 Ashique KT, Kaliyadan F, Aurangabadkar SJ. Clinical photography in dermatology using smartphones: an overview. Indian Dermatol Online J. 2015 May-Jun;6(3):158-63.

21 Quigley EA, Tokay BA, Jewell ST, Marchetti MA, Halpern AC. Technology and technique standards for camera-acquired digital dermatologic images: a systematic review. JAMA Dermatol. 2015 Aug;151(8):883-90.

22 Caffery LJ, Clunie D, Curiel-Lewandrowski C, Malvehy J, Soyer HP, Halpern AC. Transforming dermatologic imaging for the digital era: metadata and standards. J Digit Imaging. 2018 Aug;31(4):568-77.

23 Janda M, Loescher LJ, Soyer HP. Enhanced skin self-examination: a novel approach to skin cancer monitoring and follow-up. JAMA Dermatol. 2013 Feb;149(2):231-6.

24 Yung A. Image acquisition in dermatology. DermNet NZ; 2021. https://dermnetnz.org/ topics/image-acquisition-in-dermatology/.

25 Hexsel D, Hexsel CL, Dal'Forno T, Schilling de Souza J, Silva AF, Siega C. Standardized methods for photography in procedural dermatology using simple equipment. Int J Dermatol. 2017 Apr;56(4):444-51.

26 Nayler JR. Clinical photography: a guide for the clinician. J Postgrad Med. 2003 Jul-Sep; 49(3):256-62. 